\title{
Influence of Thickness and Interface on the Low-Temperature Enhancement of the Spin Seebeck Effect in YIG Films
}

\author{
Er-Jia Guo, ${ }^{1,2}$ Joel Cramer, ${ }^{1}$ Andreas Kehlberger, ${ }^{1}$ Ciaran A. Ferguson, ${ }^{3}$ Donald A. MacLaren, ${ }^{3}$ \\ Gerhard Jakob, ${ }^{1}$ and Mathias Kläui, ${ }^{1, *}$ \\ ${ }^{1}$ Institute of Physics, Johannes Gutenberg-University Mainz, 55099 Mainz, Germany \\ ${ }^{2}$ Quantum Condensed Mater Division, Oak Ridge National Laboratory, Oak Ridge, Tennessee 37830, USA \\ ${ }^{3}$ SUPA, School of Physics and Astronomy, University of Glasgow, G12 8QQ Glasgow, United Kingdom \\ (Received 28 August 2015; revised manuscript received 1 June 2016; published 27 July 2016)
}

The temperature-dependent longitudinal spin Seebeck effect (LSSE) in heavy metal (HM) $/ \mathrm{Y}_{3} \mathrm{Fe}_{5} \mathrm{O}_{12}$ (YIG) hybrid structures is investigated as a function of YIG film thickness, magnetic field strength, and different HM detection materials. The LSSE signal shows a large enhancement with reductions in temperature, leading to a pronounced peak at low temperatures. We find that the LSSE peak temperature strongly depends on the film thickness as well as on the magnetic field. Our result can be well explained in the framework of magnon-driven LSSE by taking into account the temperature-dependent effective propagation length of thermally excited magnons in the bulk of the material. We further demonstrate that the LSSE peak is significantly shifted by changing the interface coupling to an adjacent detection layer, revealing a more complex behavior beyond the currently discussed bulk effect. By direct microscopic imaging of the interface, we correlate the observed temperature dependence with the interface structure between the YIG and the adjacent metal layer. Our results highlight the role of interface effects on the temperature-dependent LSSE in HM/YIG system, suggesting that the temperature-dependent spin current transparency strikingly relies on the interface conditions.

\section{INTRODUCTION}

The spin Seebeck effect (SSE) describes the phenomenon that spin currents can be thermally excited by a temperature gradient across a magnetic material [1-3]. These spin currents can be injected into an adjacent heavy metal (HM) layer with a high spin-orbit coupling, which allows one to electrically detect the spin currents due to the inverse spin Hall effect (ISHE) [1-9]. The SSE has been observed not only in metals [1] and semiconductors [2], but also in magnetic insulators [3,6-18]. Recently, related effects have also been observed in paramagnetic and antiferromagnetic materials [19-21]. Thus, the SSE effect is relevant for a large class of materials and it is of key importance to determine the effects of the spin current generated in the bulk of a material and the spin transport across its interface. In a magnetic insulator, the excitations of the localized spin-polarized electrons are the primary source of spin currents due to the absence of itinerant electrons. The injected thermal energy can propagate only through quasiparticle excitations of either the

\footnotetext{
*Author to whom all correspondence should be addressed. klaeui@uni-mainz.de

Published by the American Physical Society under the terms of the Creative Commons Attribution 3.0 License. Further distribution of this work must maintain attribution to the author $(s)$ and the published article's title, journal citation, and DOI.
}

magnetization (magnons) or of the real space atom displacements (phonons). It is crucial to have a thorough understanding of relevant interactions between magnons and phonons and the corresponding length scales that govern magnon transport. The first report of a giant enhancement of transverse SSE (TSSE) in $\mathrm{LaY}_{2} \mathrm{Fe}_{5} \mathrm{O}_{12}$ at low temperature was published by Adachi et al. [10]. However, results of the TSSE have been controversial as, for instance, in Ref. [22] the signals were claimed to be dominated by the anisotropic magnetothermopower. No experimental method has been capable of directly observing this temperature difference of magnons and phonons so that the origin of the TSSE signals, particularly over long length scales, is still unclear. In particular, it is challenging to generate only pure in-plane thermal gradients without out-of-plane components, which then lead to parasitic effects. Therefore, most recent studies have focused on the longitudinal configuration where a low-temperature enhanced SSE signal was also observed in $\mathrm{Y}_{3} \mathrm{Fe}_{5} \mathrm{O}_{12}$ (YIG) single crystals [12]. However, there is no consensus on the explanation based on magnon-mediated theory [11] as well as a phonon-drag SSE scenario proposed to explain the experimental results at low temperature. Similar to the previously known phonon-drag for the conventional Seebeck effect, a temperature dependent phonon lifetime can play a role, as it reaches a maximum at low temperatures, leading to a peak of the thermal conductivity similar to the peak observed for the SSE. Based on this 
observation, a strong interaction between phonons and magnons was proposed with the phonons "dragging" the thermally excited magnons, thereby enhancing the pumped spin current into the detection layer. The phonon-magnon coupling has thus been suggested to explain the observed enhancement of the SSE signal at low temperatures [11-14]. Recent measurements of the temperature-dependent thermal conductivity of YIG single crystals show that the phonon contribution to the thermal conductivity reaches its maximum at $\sim 25 \mathrm{~K}$ [14], which is $\sim 50 \mathrm{~K}$ lower than the observed low-temperature SSE peak [10,12]. Additionally, the magnon contribution to the thermal conductivity has been found to be less than $5 \%$ at $8 \mathrm{~K}$ and decreases rapidly with increasing temperatures [14]. As in insulating ferromagnets, the main decay channel of angular momentum is through magnon-phonon interactions. Without magnon conservation, one can deduce from the low damping of YIG a weak coupling between the magnons and phonons. In fact, the observed transport of magnons over a long distance in magnetic insulators also supports the notion of a relatively weak interaction with phonons and impurities [3,13]. Therefore the validity of a phonon-drag SSE mechanism that uses strong phonon-magnon interactions to explain YIG's SSE temperature dependence is unclear. The genuine origin of the low temperature SSE enhancement needs to be clarified.

In addition to these discrepancies, the SSE signal is indirectly probed via the ISHE so that not only the bulk properties of magnetic materials can be important, but the injection efficiency of the spin current across the interface is also crucial. As recently observed, for metallic interfaces between a metallic ferromagnet and a nonmagnetic metal layer, interface effects can play a crucial role in determining the spin current effects and dominate the measured signals [23-26]. However, the interfaces between metals are different from insulator-metal interfaces where coupling tends to be more localized. Although epitaxial growth of detection layers with large spin Hall angles on oxides, like YIG, are challenging, previous work has shown that the SSE signal can be enhanced when an atomically smooth interface with a preferred orientation is introduced [16-18]. At room temperature, it is known that different detection materials that possess different spin Hall angles can lead to different SSE signal amplitudes [6]. However, the process of spin transmission across the interface and, in particular, the temperature-dependent spin current transparency of interfaces, needs to be ascertained together with the atomistic interface structure to understand to what extent the interfaces play a role for the temperature dependence of the SSE.

In this paper, we study the temperature and magnetic field dependence of the longitudinal SSE (LSSE) in a YIG single crystal and thin films ranging from $150 \mathrm{~nm}$ to $50 \mu \mathrm{m}$ in thickness. A giant enhancement of the LSSE signal is observed at low temperatures. We find that this LSSE peak shifts to a higher temperature as the film thickness decreases, and it shifts towards lower temperatures with increasing magnetic field. A simple phenomenological model is introduced to explain the observed thickness and field dependence by considering the temperaturedependent effective magnon propagation length. Surprisingly, we find a strong dependence of the peak temperature on the ISHE detection material, revealing that the temperature-dependent LSSE signal not only depends on the bulk thermal spin current, but also strongly relies on the interface coupling to the detection material, which has not been taken into account previously. By direct spectroscopic imaging we determine the interface structure and find that the interface termination is correlated with the measured temperature dependence of the signal.

\section{EXPERIMENTAL PROCEDURE}

The samples we use in the present study are YIG thin films grown on a (111)-oriented $\mathrm{Gd}_{3} \mathrm{Ga}_{5} \mathrm{O}_{12}$ (GGG) substrate $\left(5 \times 10 \times 0.5 \mathrm{~mm}^{3}\right)$ by liquid phase epitaxy (LPE) and a (111)-oriented YIG slab $\left(5 \times 10 \times 1 \mathrm{~mm}^{3}\right)$. The thickness of YIG thin films ranges from $150 \mathrm{~nm}$ to $50 \mu \mathrm{m}$. The surface characteristics of the YIG samples and interface qualities are carefully checked by atomic force microscopy (AFM) and x-ray reflectivity (XRR) (see Appendix A). The rms roughness of all the YIG samples is less than $5 \AA$, providing a smooth surface for the further growth of the HM detection layer. Before the HM layer deposition, the YIG surface is treated by in situ heating and then followed by argon plasma milling in order to obtain a clean interface. A thin HM layer is sputter deposited on top of the YIG samples and then patterned into strips by optical lithography and ion beam etching. All the strips have the same geometry with a length of $L=4 \mathrm{~mm}$ and a width of $w=100 \mu \mathrm{m}$. Our LSSE measurements are carried out in the longitudinal configuration with the temperature gradient parallel to the surface normal ( $z$ axis) [7]. The samples are sandwiched between a resistive heater and a thermal sensor, and further mounted on a copper heat sink using thermally conducting grease. The temperature-dependent measurements are performed in a cryostat with a variable temperature insert. The thermal gradient can be varied by changing the heat currents $\left(I_{\text {heat }}\right)$ of the resistive heater (for more details, see Appendix B). The in-plane magnetic field $(H)$ is applied along the $y$ axis, perpendicularly oriented to the long axis of the strips ( $x$ axis) in order to provide maximum signals due to the ISHE. $H$ is strong enough to ensure full alignment of the magnetization in the YIG. The LSSE signal can be detected by measuring the voltage drop at the two ends of the strip. We use both the magnetic fieldsweep method and the temperature-sweep method to record the LSSE signals. The former method has been commonly used in previous reports [5-13]. The LSSE voltages flip sign when the direction of magnetic field reverses, allowing us to extract the $V_{\text {LSSE }}$ from the difference between two 
saturated LSSE signals; hence, $V_{\mathrm{LSSE}}$ is calculated by $V_{\mathrm{LSSE}}=\left[V_{\mathrm{LSSE}}(+H)-V_{\mathrm{LSSE}}(-H)\right] / 2$. The latter method is to keep the magnetic field as a constant while the temperature is swept twice (first at $+H$, then at $-H$ ). The $V_{\mathrm{LSSE}^{-}}-T$ curves are obtained by extracting the differences between $V_{\mathrm{LSSE}}(+H)-T$ and $V_{\mathrm{LSSE}}(-H)-T$ curves. We keep an ultraslow sweep rate with a temperature change of $-0.25 \mathrm{~K} / \mathrm{min}$. This method provides sufficient time for the thermal equilibration between sample and cryostat during the temperature drop, yielding reproducible measurement conditions. In order to quantitatively compare the LSSE signals between different samples, the LSSE coefficients are calculated by $\sigma_{\mathrm{LSSE}}=\left(V_{\mathrm{LSSE}} / \Delta T\right) \times\left(L_{z} / L\right)$, where $L_{z}$ is the total thickness of the YIG slab or YIG/GGG samples. Transmission electron microscopy is conducted on a JEOL ARM200cF instrument, operated at $200 \mathrm{kV}$ and equipped with a cold field emission gun. Thin lamella samples are prepared for TEM characterization on an FEI Nova Dualbeam focused ion beam system using standard "lift-out" protocols. Electron energy loss spectroscopy (EELS) and energy dispersive x-ray spectroscopy (EDS) are conducted on a Gatan Quantum spectrometer and Bruker $X$-Flash spectrometer, respectively. The latter are used for Pt mapping. Elemental maps are acquired using a combination of the spectrum imaging [27] and dual EELS [28] methodologies.

\section{RESULTS AND DISCUSSION}

Figure 1(a) shows the temperature-dependent $\sigma_{\text {LSSE }}$ for a $\mathrm{Pt} /$ YIG slab. The LSSE signal gradually increases with a temperature decrease and exhibits a pronounced peak at around $75 \mathrm{~K}$, which is qualitatively consistent with previous reports $[10,12]$. We also repeat the LSSE measurements by sweeping the magnetic field at fixed temperatures. The results obtained by the field-sweep method are shown as open symbols in Fig. 1(a). The good agreement between two measuring methods validates the reproducibility and reliability of the temperature-sweep results, which we use for all further measurements in this work. Figure 1(b) shows the temperature-dependent $\sigma_{\mathrm{LSSE}}$ for $\mathrm{Pt} / \mathrm{YIG}$ samples with YIG thicknesses ranging from $150 \mathrm{~nm}$ to $50 \mu \mathrm{m}$. The low-temperature enhancement of the LSSE is observed for thicker YIG films and the amplitude of the LSSE peak is reduced as the film thickness decreases. The low-temperature LSSE peak positions are obtained from the Gaussian fits of each $\sigma_{\mathrm{LSSE}}-T$ curve. We find there is no visible peak for the films with a thickness below $600 \mathrm{~nm}$. The LSSE peak shifts to higher temperatures [shown in the inset of Fig. 1(a)] and its width increases as the film thickness is reduced. To exclude any possible artifacts involved in the LSSE measurements, additional effects, which exhibit temperature dependences and may influence the LSSE peak positions, are analyzed. The temperature-dependent resistances of $\mathrm{Pt}$ strips are monitored simultaneously during the measurements.

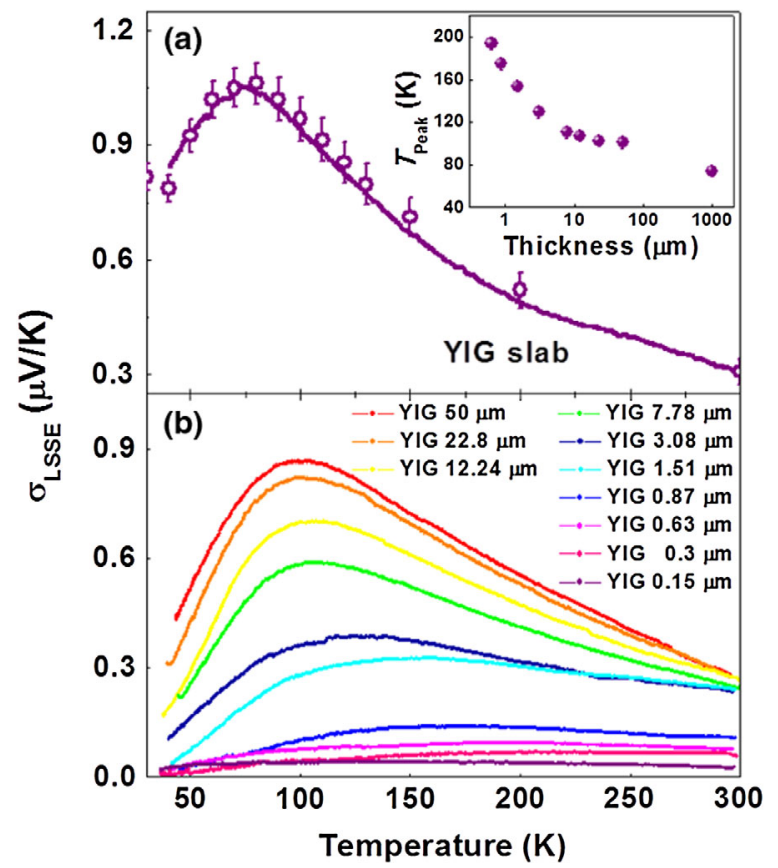

FIG. 1. Temperature-dependent LSSE coefficients $\left(\sigma_{\mathrm{LSSE}}\right)$ of (a) a YIG slab and (b) YIG thin films with a wide range of thicknesses. The open symbols in (a) represent the results recorded by field-sweep mode measurements. The inset of (a) shows the peak position of the $\sigma_{\mathrm{LSSE}}-T$ curves as a function of film thickness.

The resistivity $\left(R_{\mathrm{Pt}}\right)$ of the as-deposited $\mathrm{Pt}$ layer is $\sim 5 \times 10^{-5} \Omega \mathrm{cm}$ at room temperature and shows a monotonic reduction as the temperature decreases. We calculate $I_{\mathrm{LSSE}}\left(=V_{\mathrm{LSSE}} / R_{\mathrm{Pt}}\right)$, yielding a shift of the peak temperature of $\sim 5 \mathrm{~K}$, which is identical for all samples due to the similar nature of the metal stripes. Therefore, the remaining mechanisms responsible for the strong temperature dependence of the LSSE are the bulk effects in the YIG and the interface effects with the adjacent HM layers.

In the HM/YIG/GGG system, the total thermal conductivity is dominated by the YIG film and GGG substrate due to the much smaller thickness and higher thermal conductivity of the HM detection layer. We do not find a significant difference in the (perpendicular-to-the-plane) thermal conductivity for a HM/YIG/GGG system for different metals because the thermal transport is limited by the YIG/GGG $[29,30]$. This means that the thermal gradient across the HM/YIG, which governs the thermally generated spin current, is not significantly impacted by the metal detection layer. In the YIG bulk material, phonons coexist with magnons and contribute to the thermal conductivity at all temperatures. Our recent measurements of the thermal conductivity as a function of temperature on these YIG samples reveal a minor shift of the peak position of the thermal conductivity with decreasing film thickness [30]. The absence of the pronounced thickness dependence of the thermal conductivity peak in combination with the large mismatch between both peak 
temperatures suggests a less important contribution of the phonon-magnon interaction than previously assumed for the transverse SSE in YIG. In other words, the strength of phonon-magnon coupling for the probed thermally excited magnons involved in our LSSE experiments is not strong enough to dominate the temperature dependence. Therefore, a more apt approach to explain the lowtemperature enhancement of the LSSE is to consider the concept of a magnonic spin current alone, based on the idea of a temperature-dependent effective thermal magnon propagation length that describes the propagation length of the combined thermally generated spin current and the detection mechanism of the spin current that is transmitted across the interface and detected by the ISHE [7,31]. In our previous work, we demonstrated that the thermally excited magnons that contribute to the ISHE signals exhibit a finite propagation length [8]. This behavior is well described by an atomistic spin model, which is capable of describing a thermally excited magnon accumulation generated by the temperature gradient using $[7,31]$

$$
V_{\mathrm{LSSE}} \propto 1-\exp (-L / \xi)
$$

where $\xi$ is the characteristic length of the LSSE, regarding it as the effective propagation length of all thermally excited magnons, which arrive at the adjacent detection layers. Figures 2(a)-2(c) present the $\sigma_{\text {LSSE }}$ as a function of film thickness at 300, 200, and $120 \mathrm{~K}$. The $\sigma_{\mathrm{LSSE}}$ increases gradually with increasing thickness and saturates above a critical thickness. The results are in good agreement with our previous findings [7], indicating that only magnons excited at distances smaller than $\xi$ from the interface can contribute to the detected ISHE signals. By fitting our results with Eq. (1), we can estimate $\xi$ across the whole temperature range. The results are shown in Fig. 2(d). We find a monotonic increase of $\xi$ with decreasing temperature. By fitting a functional dependence of $\xi \propto T^{-n}$, we obtain

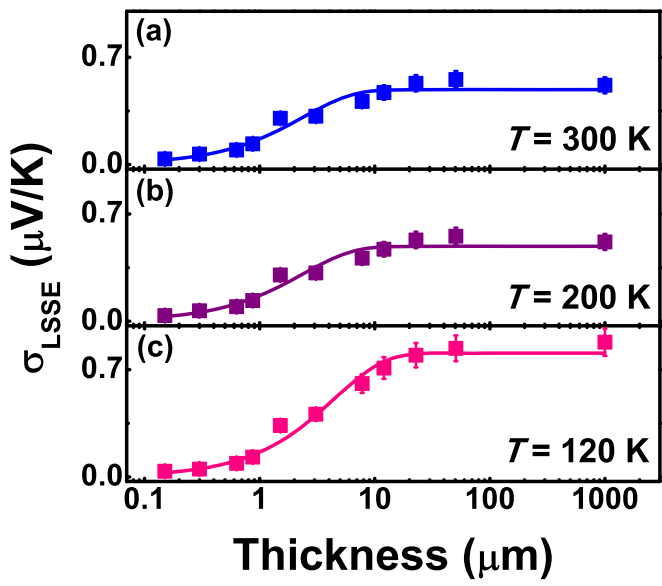

an exponent $n=0.97 \pm 0.02$, which is close to 1 , a dependence expected, for instance, for inelastic scattering of bosonic quasiparticles. Ritzmann and co-workers [31,32] have shown that $\xi$ is inversely proportional to the Gilbert damping constant $\alpha\left(\xi \sim \alpha^{-1}\right)$, revealing a longer propagation length with a lower magnetic damping. Previously, using a ferromagnetic resonance (FMR) setup, Jungfleisch et al. [33] had determined that the Gilbert damping parameter of the LPE YIG films is on the order of $10^{-4}$ at room temperature. Note that FMR probes only the zone center magnons, which may not be the only ones involved in LSSE. However, the FMR results indicate the high quality of the material and the resulting low damping of LPE grown YIG films [33]. Additionally, Spencer and LeCraw [34] have shown that the magnetic damping constant $\alpha$ of a YIG single crystal depends approximately linearly on the temperature where our LSSE experiments are carried out (with slight variations due to the inherent microstructure defects). While the applicability of this temperature dependence for all thin films grown by different methods is not obvious, such a linear temperature dependence of the Gilbert damping in combination with its inverse proportionality leads to the observed $\xi \sim T^{-1}$ dependence, which agrees well with our experiments [Fig. 2(d)].

Having understood the origin of the temperature dependence of $\xi$, we can use this concept to explain the temperature dependence of the LSSE signals. For thick YIG films, $\xi$ increases with decreasing temperature, so that more magnons can reach the detection layer, leading to an enhancement of the LSSE signal. In parallel, the total number of thermally excited magnons decreases as a function of temperature, which can be best approximated using the specific heat of the magnetic lattice. At a certain temperature, the increase of $\xi$ is limited by the combined boundary scattering and magnon-phonon scattering mechanisms. At this temperature, $\sigma_{\text {LSSE }}$ reaches a maximum

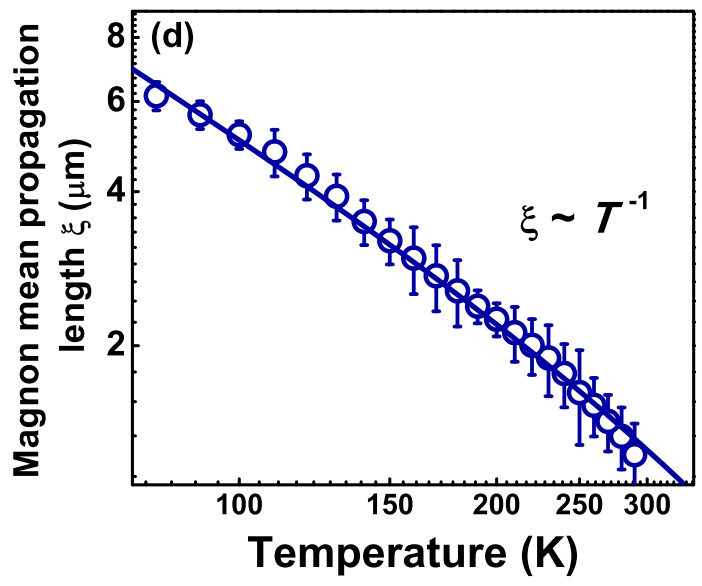

FIG. 2. Thickness-dependent $\sigma_{\text {LSSE }}$ measured at a fixed temperature of (a) $300 \mathrm{~K}$, (b) $200 \mathrm{~K}$, and (c) $120 \mathrm{~K}$. The solid lines are the fitting results according to the atomistic spin model. (d) Temperature-dependent effective magnon propagation length $\xi$. The solid line is the fit to the power law of $\xi \sim T^{-n}$, where the fitted exponent $n \sim 1$. 
since the total number of magnons that can reach the $\mathrm{Pt}$ layer cannot increase further. At even lower temperature, $\xi$ stays constant, while the amount of thermally excited magnons decreases, leading to a decrease of $\sigma_{\mathrm{LSSE}}$ as the temperature decreases. Since $\xi$ is limited in the thinner YIG films at higher temperatures, the peak temperature is shifted towards higher temperatures as film thickness decreases and the overall amplitude of the LSSE signal decreases as well. For YIG films showing an absence of a peak, $\xi$ is of the order of the film thickness at room temperature, as shown in Fig. 2(d), leading to a temperature dependence of the $\sigma_{\text {LSSE }}$ dominated by the total amount of thermal magnons in the system. Since the excitation of thermal magnons follows a Bose-Einstein distribution, it is smeared out for higher temperatures, leading to a broadening of the LSSE peak. This combination of a temperature-dependent $\xi$ and the total number of thermal magnons can phenomenologically explain the presence of the LSSE peak and its thickness dependence. One needs to be cautious when comparing the propagation length of thermal magnons in our work and what is sometimes called magnon spin relaxation length. For instance, the "propagation length" in the works of Cornelissen and co-workers $[35,36]$ is a result of a measurement where the thermal magnons are generated at a fixed position, propagate in the ferromagnetic insulator, and are then detected at a certain distance. Only low-energy magnons which have long propagation length will be measured, while the high-energy magnons are damped out over the long distance. In our work, the generated thermal magnons of all energies arrive in the adjacent detection layer and contribute to the LSSE signal. Therefore, the effective propagation length we discuss in our work is a value that results from the whole magnon spectrum probed here and which is distinct from measurements in other geometries.

Applying a high magnetic field can suppress low-energy magnonic excitations and thus provide a possible way to manipulate the magnon distribution in magnetic insulators $[14,37,38]$. In order to rule out any possible proximity effects $[8,39]$, the temperature-dependent LSSE measurements are carried out using a Pt/Cu/YIG(50 $\mu \mathrm{m})$ sample by inserting an ultrathin $\mathrm{Cu}$ interlayer with a thickness of $2 \mathrm{~nm}$ between Pt and YIG. Figure 3(a) shows the field dependence of the LSSE for Pt/Cu/YIG(50 $\mu \mathrm{m})$ at various temperatures. The field suppression ratio $\Delta \sigma_{\mathrm{LSSE}}[=[1-$ $\left.\left.\sigma_{\mathrm{LSSE}}(9 \mathrm{~T}) / \sigma_{\mathrm{LSSE}}(0.2 \mathrm{~T})\right] \times 100 \%\right]$ drops monotonically from $\sim 30 \%$ at room temperature to $\sim 9 \%$ at $30 \mathrm{~K}$, as illustrated in Fig. 3(b). The temperature-dependent LSSE under variable magnetic fields from 0.2 to $9 \mathrm{~T}$ is shown in Fig. 3(c). We find a significant reduction of $\sigma_{\mathrm{LSSE}}$ in an applied magnetic field at high temperatures, and the reduction becomes smaller with decreasing temperature, in agreement with the field-sweep measurements shown in Fig. 3(b). Increasing the magnetic field also leads to a shift of the LSSE peak of $\sim 10 \mathrm{~K}$ towards lower temperatures. A possible magnetoresistance effect of the Pt detector and heater layers is monitored simultaneously during the LSSE measurements. The field-induced resistance changes are less than $0.04 \%$, indicating a negligible impact on the observed field suppression of the LSSE signals. We find that our results cannot be explained by the opening of an energy gap for the thermally excited magnons (energy of the order of $\sim \mathrm{kT}$ ), since our field suppression of the LSSE signal is observed even at room temperature, which is much
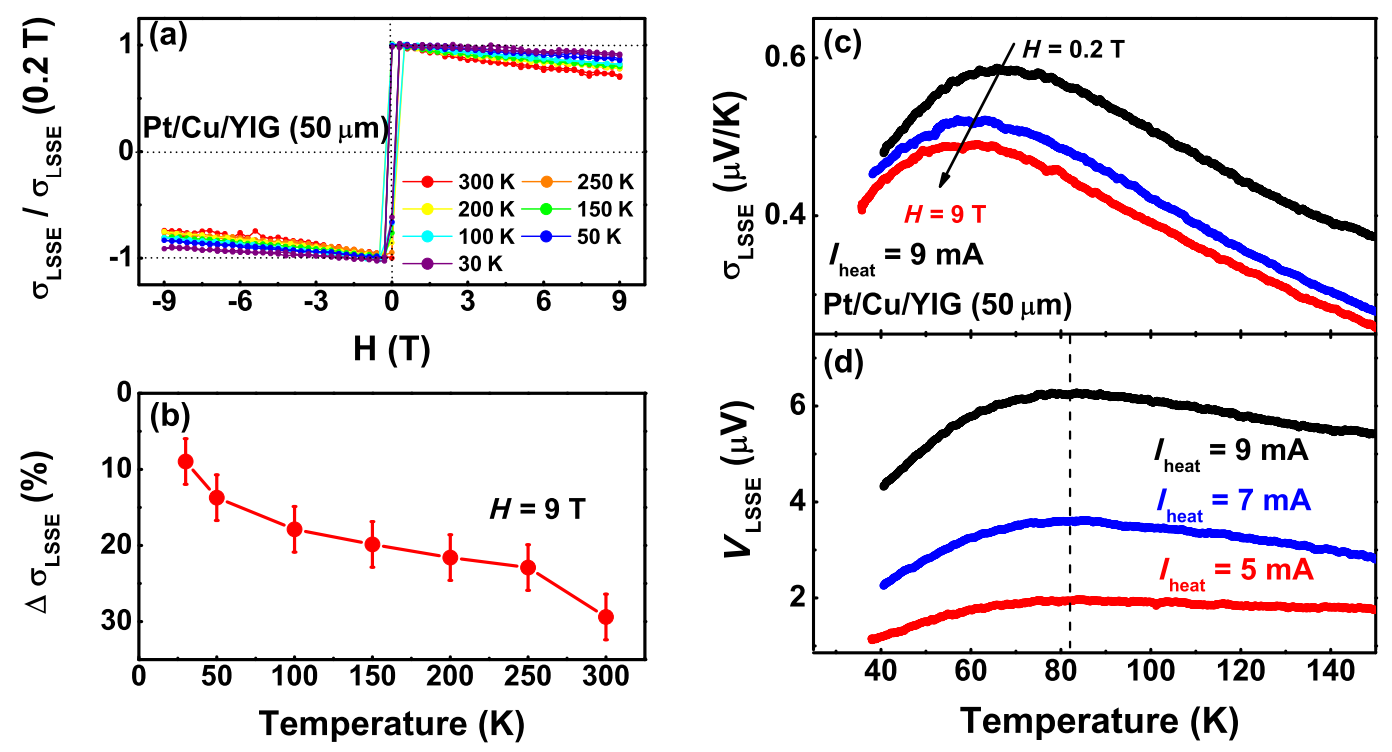

FIG. 3. (a) Magnetic field-dependent normalized LSSE signals of $\mathrm{Pt} / \mathrm{Cu} / \mathrm{YIG}$ hybrids at various temperatures from $30 \mathrm{~K}$ to room temperature. (b) Temperature-dependent field suppression ratio of $\mathrm{Pt} / \mathrm{Cu} / \mathrm{YIG}$ hybrids at $9 \mathrm{~T}$. (c) Temperature-dependent $\sigma_{\mathrm{LSSE}}$ of $\mathrm{Pt} / \mathrm{Cu} /$ YIG hybrids measured at a fixed heating power and varied magnetic fields from 0.2 to $9 \mathrm{~T}$. (d) Temperature-dependent LSSE voltages of $\mathrm{Pt} / \mathrm{Cu} / \mathrm{YIG}$ hybrids with a fixed magnetic field of $\pm 0.2 \mathrm{~T}$ and different heating currents. 
higher than the predicted magnon gap opening temperature of $\sim 12 \mathrm{~K}$ at $9 \mathrm{~T}$ [38]. Therefore, we explain our observations based on the above-used temperature-dependent $\xi$. As we know, the low-frequency magnons dominate the LSSE signal and are sensitive to the applied magnetic fields [14]. These magnons are partially frozen out by the high magnetic fields, resulting in a reduction of $\xi$. Therefore, the field suppression of the LSSE can be observed even at high temperatures. Recently, Ritzmann et al. [32] have reported a larger field suppression of the LSSE in the thicker YIG films, whereas the LSSE signal of the YIG films with thicknesses below $\xi$ is barely changed by an applied magnetic field. This thickness-dependent field suppression of the LSSE is in line with our results and interpretation. $\xi$ increases as the temperature decreases, as shown in Fig. 2(d). This temperature-induced enhancement of $\xi$ counteracts the reduction of $\xi$ caused by the high magnetic fields. As a result of these two competing factors, the field suppression of the LSSE signals is less pronounced for lower temperatures. The reduction of $\xi$ becomes larger with higher magnetic fields, thus it counteracts the low-temperature $\xi$ enhancement more effectively, leading to a smaller $\Delta \sigma_{\mathrm{LSSE}}$ at low temperature and a shift of LSSE peak towards lower temperature. At very low temperatures, $\xi$ stays constant and therefore the suppression of the SSE signal amplitude is mainly caused by the magnetic field-induced reduction of $\xi$, as reported in Refs. [37,38]. Figure 3(d) shows the temperaturedependent $V_{\text {LSSE }}$ for different heating powers. We find that the LSSE peak temperatures stay constant, indicating that the intrinsic $\xi$ is barely changed by altering $\Delta T$. The magnitude of $V_{\text {LSSE }}$ increases linearly with increasing $\Delta T$, since the $\Delta T$ applied here is small so that the LSSE stays in a linear response regime [40]. Our results thus demonstrate the validity of our finite effective magnon propagation length model, which holds for all thermally excited magnons accessible by the applied temperature gradients.

Having determined the effects resulting from the bulk magnonic spin current, we next investigate possible previously neglected interface effects on the temperaturedependent LSSE in the HM/YIG system. In addition to the $\mathrm{Pt} / \mathrm{YIG}$, we now introduce $\mathrm{Pd}$ and $\mathrm{W}$ as spin detection layers for comparison. A thin Pd and $\mathrm{W}$ layer is sputtered on the YIG surface (for more details, see Appendix A). The thickness of the Pd and W layer is set to be the same as for $\mathrm{Pt}(\sim 5 \mathrm{~nm})$. After $\mathrm{W}$ is sputtered on YIG, it is later covered by a thin $\mathrm{AlO}_{x}$ layer to prevent any oxidization of $\mathrm{W}$ in the ambient condition. The YIG films with a thickness of $50 \mu \mathrm{m}$ are identical, taken from the same wafer to exclude any variations from bulk effects. Figure 4(a) shows a comparison of the temperature dependence of $\sigma_{\mathrm{LSSE}}$ in $\mathrm{Pt} / \mathrm{YIG}, \mathrm{Pd} / \mathrm{YIG}$, and W/YIG hybrids. The $\sigma_{\mathrm{LSSE}}$ of the W/YIG hybrid shows a negative LSSE signal due to the negative value of the spin Hall angle of $\mathrm{W}\left(\theta_{\mathrm{SHE}, \mathrm{W}}=-0.14\right)$ [41]. Compared to $\mathrm{Pt} / \mathrm{YIG}, \sigma_{\mathrm{LSSE}}$ of $\mathrm{Pd} / \mathrm{YIG}$ is much smaller than that of Pt/YIG. This can be quantitatively understood due to the smaller spin Hall angle $\left(\theta_{\text {SHE,Pd }}=0.01\right)$ and resistivity $\left(\rho_{\mathrm{Pd}}=0.25 \mu \Omega \mathrm{m}\right)$ of $\mathrm{Pd}$ compared to that of $\mathrm{Pt}\left(\theta_{\mathrm{SHE}, \mathrm{Pt}}=0.068, \rho_{\mathrm{Pt}}=0.42 \mu \Omega \mathrm{m}\right)$ $[42,43]$. We now compare the LSSE peak positions and find that it varies strongly from $100 \mathrm{~K}$ in Pt/YIG to $56 \mathrm{~K}$ in W/ YIG and to $49 \mathrm{~K}$ in Pd/YIG. To understand the observation, several possible mechanisms related to interface effects that underlie the temperature-dependent LSSE are discussed. This difference could, firstly, be intuitively attributed to a difference in the temperature dependence of the spin Hall angle $\left(\theta_{\mathrm{SHE}}\right)$ of the three HM layers. Tang et al. [44] report that $\theta_{\mathrm{SHE}}$ of Pd approximately linearly increases from 0.01 at room temperature to 0.02 at $130 \mathrm{~K}$. On the other hand, $\theta_{\mathrm{SHE}}$ of $\mathrm{Pt}$ and $\mathrm{W}$ barely changes across the whole temperature range [41-43]. Secondly, the spin diffusion lengths $\left(\lambda_{\mathrm{SD}}\right)$ of the detection layers can be temperature dependent. The spin-injection efficiencies across the HM/YIG interfaces strongly depend on the backflow spin currents induced by the spin accumulation in the HM layers. These effects become more pronounced at low temperatures as $\lambda_{\mathrm{SD}}$ increases with decreasing temperature [42-48]. This could explain the observed detection material
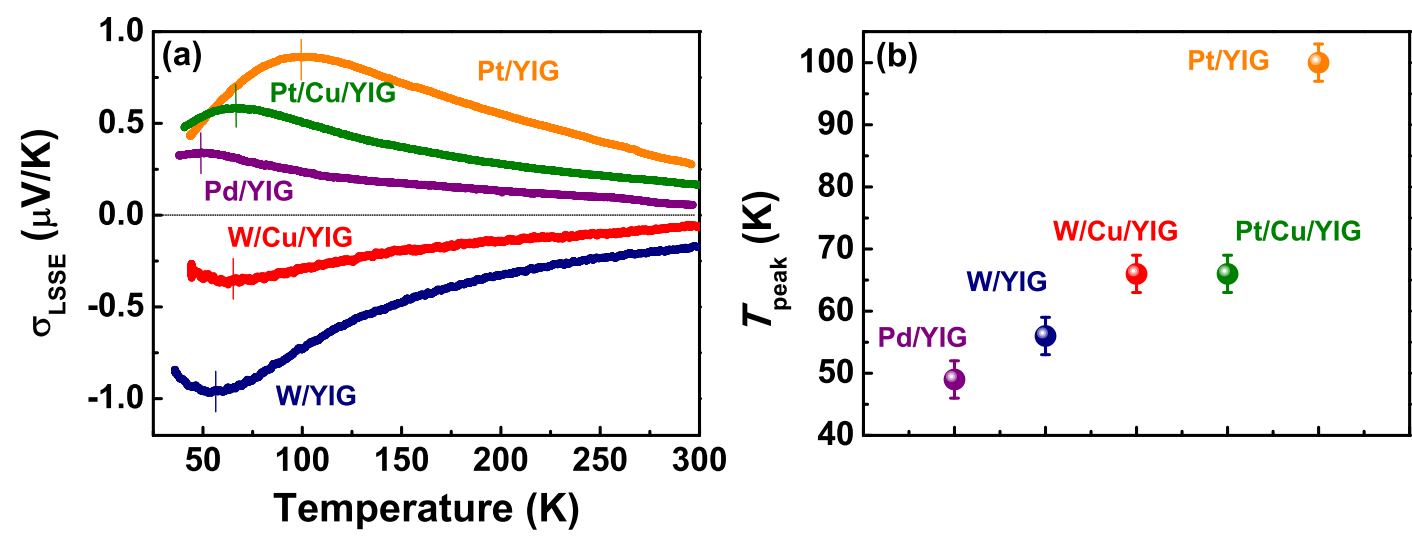

FIG. 4. (a) Temperature-dependent $\sigma_{\mathrm{LSSE}}$ measured at Pt/YIG, Pd/YIG, W/YIG, Pt/Cu/YIG, and W/Cu/YIG hybrids. The thickness of YIG films is $50 \mu \mathrm{m}$. (b) The peak positions of the $\sigma_{\mathrm{LSSE}}-T$ curves measured for YIG films with different capping layers. 
dependence of the LSSE, as the temperature dependences of $\lambda_{\mathrm{SD}}$ for different detection layers are found to be different. For Pt, $\lambda_{\mathrm{SD}}$ is $2.0 \pm 2.2 \mathrm{~nm}$ at $300 \mathrm{~K}$ and slightly increases to $3.4 \pm 0.3 \mathrm{~nm}$ at $10 \mathrm{~K}$ [43]; for $\mathrm{Pd}, \lambda_{\mathrm{SD}}$ is $\sim 9 \mathrm{~nm}$ at $300 \mathrm{~K}$ and increases to $\sim 25 \mathrm{~nm}$ at $4.2 \mathrm{~K} \mathrm{[45];}$ while for $\mathrm{W}, \lambda_{\mathrm{SD}}$ is found to be $2.1 \mathrm{~nm}$ at room temperature and doubles its value to $4.8 \mathrm{~nm}$ at $4.2 \mathrm{~K}[41,43]$. Thus, the change of the temperature dependence of the spin-injection efficiency induced by different detection layers may affect the temperature dependence and the peak positions of the LSSE signals.

To check this possibility, we insert a $\mathrm{Cu}$ layer with a thickness of $2 \mathrm{~nm}$ between the $\mathrm{Pt}(\mathrm{W})$ and the YIG. We see that the $\sigma_{\mathrm{LSSE}}$ of $\mathrm{Pt}(\mathrm{W}) / \mathrm{Cu} / \mathrm{YIG}$ is reduced dramatically compared with that of $\mathrm{Pt}(\mathrm{W}) / \mathrm{YIG}$, as shown in Fig. 4(a). Effects of a signal reduction due to the $\mathrm{Cu}$ interlayer have been reported in both spin pumping experiments [33,49] and SSE measurements [5,7]. The inserted $\mathrm{Cu}$ between YIG and Pt partially suppresses the spin currents pumped into the Pt layer. A fraction of the accumulated spins in the $\mathrm{Cu}$ interlayer diffuse back to the YIG films or flip while the remainder is transmitted into the Pt detection layer, leading to the partial loss of the injected spin currents. Given the large spin mixing conductance of the all metal interface between $\mathrm{Cu}$ and $\mathrm{Pt}(\mathrm{W})$ and the large spin diffusion length in $\mathrm{Cu}$ [48], the total amount of reduction is surprising and can be related to the interface with the YIG film, as we discuss further below. Most importantly, however, the LSSE peak positions of the two HM/YIG structures with the $\mathrm{Cu}$ interlayer are the same, $66 \mathrm{~K}$ [Fig. 4(b)]. Furthermore, they also exhibit the same temperature dependence, which is completely different from the Pt/YIG and W/YIG stacks. This allows us to unambiguously exclude temperature effects in the detector materials (Pt and $\mathrm{W}$ ), and we can attribute the observed temperature dependence to the interface between YIG and the adjacent metals. From our results it is clear that the temperature-dependent LSSE is significantly changed by simply changing the interface in contact with the YIG. These unexpected results cannot be explained by the scenario of phonon-drag SSE due to the strong phonon-magnon interaction or any bulk effects $[10,12]$.

Finally, to obtain a microscopic understanding of the measured signals and to trace the origin of different temperature dependences, we conduct transmission electron microscopy measurements on different HM/YIG interfaces. Figures 5(a) and 5(b) show high-resolution TEM images of $\mathrm{YIG} / \mathrm{Cu} / \mathrm{Pt}$ and $\mathrm{YIG} / \mathrm{Pt}$ layer stacks, respectively. Inset into each image is a colored panel that indicates the elemental $(\mathrm{Fe}, \mathrm{Cu}, \mathrm{Pt})$ distribution within the interfaces of the two samples, derived from scanning-TEM elemental mapping using EELS and EDS. The TEM analysis indicates the metallic films to be smooth, flat, and continuous across the substrate and the YIG films to have a high-quality epitaxial and crystalline relationship to (a)

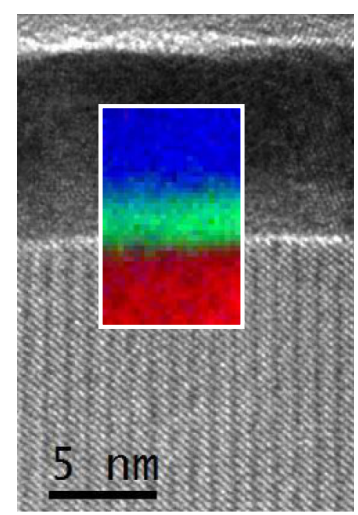

(b)

(c)

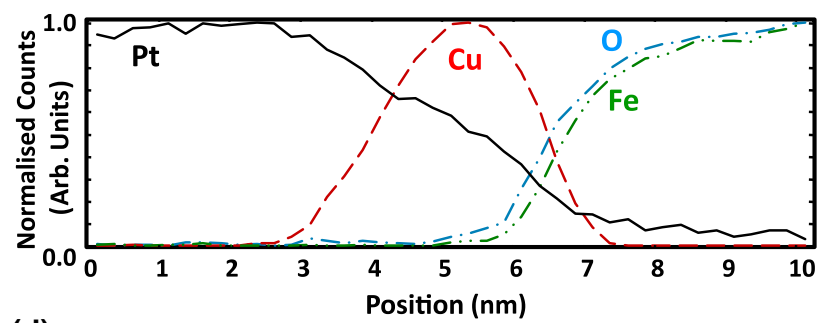

(d)
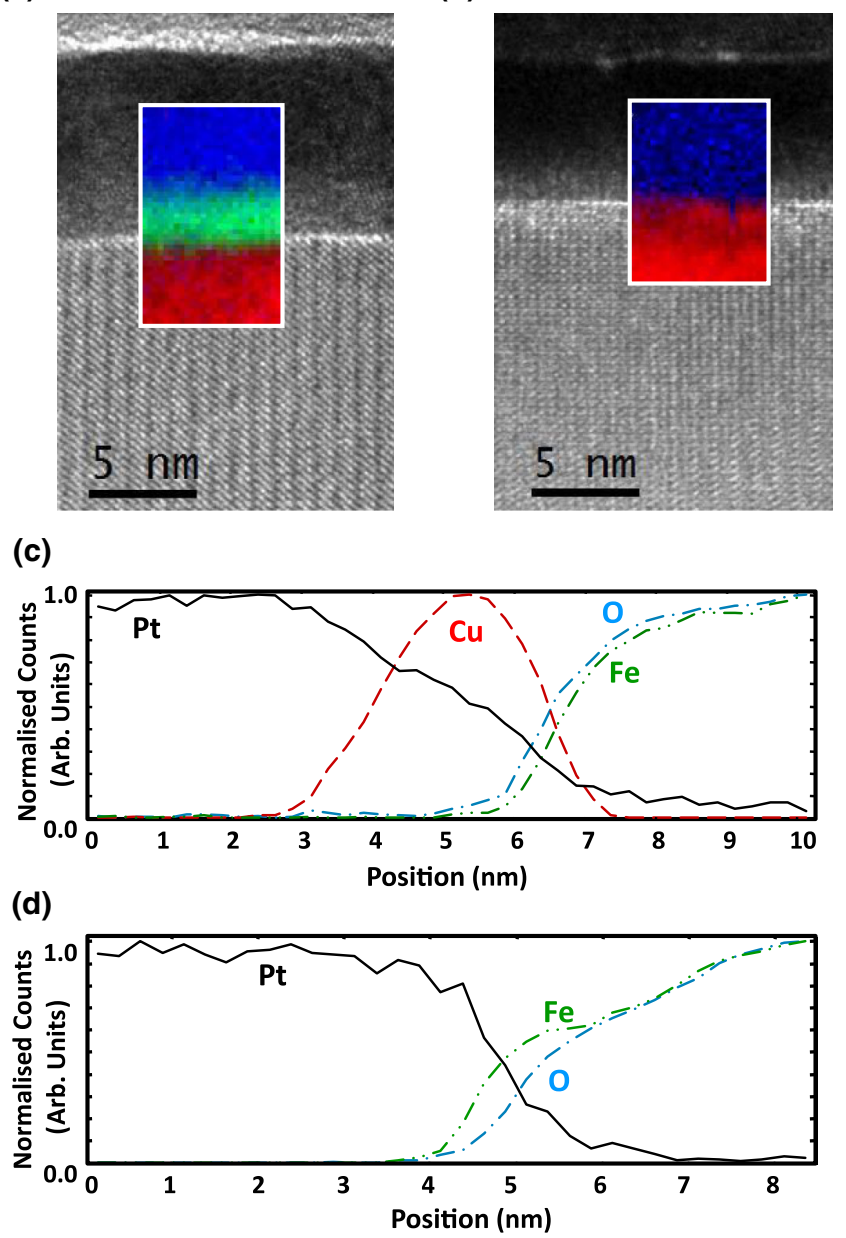

FIG. 5. Electron microscopy analysis of (a),(c) Pt/Cu/YIG and (b),(d) Pt/YIG samples. (a),(b) TEM images of the YIG (lower) and metallic layers with (inset) colored panels indicate the distributions of (red) $\mathrm{Fe}$, (green) $\mathrm{Cu}$, and (blue) Pt across the interface. Elemental maps are generated by EELS in scanningTEM mode in similar areas to those indicated. Integrated, normalized signal intensities across the data sets suggest changes in the interfacial structure (see text for details).

the substrate, without evidence of, for example, granularity, columnar or three-dimensional growth, or substantial dislocation networks. The elemental maps reveal sharp interfaces between the $\mathrm{HM}$ layer ( $\mathrm{Pt}$ or $\mathrm{Cu}$ ) and the YIG films with layer thicknesses in good agreement with XRR results. The graphs of Figs. 5(c) and 5(d) are the integrated, normalized signals across the inset panels of Figs. 5(a) and 5(b) and they suggest subtle differences in interfacial structure. In the case of $\mathrm{Pt} / \mathrm{Cu} / \mathrm{YIG}$, the iron and oxygen signals from the YIG rise in tandem, with a suggestion that the interface is oxygen terminated. The Pt trend appears to extend throughout the $\mathrm{Cu}$ layer, although we note that the data derive from noisier EDS scans and are statistically insignificant within the YIG. For Pt/YIG, on the other hand, the Fe signal of the YIG clearly rises before the oxygen signal, a trend repeated in a number of distinct data sets. 
Scanning TEM images (not shown) also indicate a slightly broader interface region between the crystalline YIG and the metal layer, perhaps because of the presence of a subnanometer region of intermixing. The experimental evidence therefore shows a difference in the interfacial structures between the HM layer and YIG films, suggesting that the different temperature dependences of the LSSE signals are influenced by interfacial effects. Furthermore, the drastic decrease of the signal due to the $\mathrm{Cu}$ interlayer can be explained by a different strength of the coupling of the magnonic spin currents in the YIG to the conduction electrons in the metal layer for YIG/Cu and YIG/Pt.

We note that the spin transmissivity probability can be spectrally dependent as well [48]. One can expect a large change of magnon transmissivity across the different interfaces when the magnon energy becomes comparable with the relevant energy scale of electrons [50]. Different interface conditions may change the spectrally dependent magnon transmissivity probability, leading to the observed interface-dependent LSSE signals. Interfacial damping may influence the spin pumping at the HM/YIG interfaces. It may play a significant role when the film is very thin. However, for our micron-range thickness films, we can ignore the influence of interfacial damping. To quantify the role of the possible sources that we identify, further work, including appropriate theoretical calculations, are necessary, which is beyond the scope of our present study. At the moment, to our best knowledge and upon consultation with leading theoretical groups, there are no experimental work or solid quantitative theoretical calculations to compare to our data. However, from the current experiment results, we can phenomenologically determine the transparency of the thermal magnons for different interfaces. We show that the larger LSSE signals and the lower LSSE peak temperatures are observed in the thicker YIG films, indicating that a larger amount of thermal magnons can reach the Pt/YIG interface and contribute to the detected ISHE voltages. In the identical YIG films, the thermally excited magnons are quantitatively the same for the same temperature gradient. Based on this analysis, we can conclude that the Pd/YIG interface, which shows the lowest LSSE peak temperature, possibly possesses the highest transmissivity for the thermal magnons which contribute to the detected LSSE signals, followed by the W/YIG, $\mathrm{Pt}(\mathrm{W}) / \mathrm{Cu} / \mathrm{YIG}$, and $\mathrm{Pt} / \mathrm{YIG}$ interfaces. Our results clearly reveal that the interfaces between adjacent layer and magnetic insulators, as an important parameter for the LSSE, can dramatically influence the temperature dependence of LSSE, which was previously ignored.

\section{CONCLUSION}

In summary, we report the temperature and thickness dependence of the LSSE for YIG single crystals and thin films with different capping layers for SSE signal detection.
Our results reveal a giant enhancement of the LSSE at low temperatures and the LSSE peak temperature is strongly dependent on the film thickness, showing a shift towards higher temperatures as the film thickness decreases. This dependence is distinct from the thermal conductivity measurements, indicating no dominating role of phononmagnon coupling for the studied LSSE. The LSSE peak temperature can be altered by the magnetic field, which causes a shift towards lower temperatures for higher magnetic fields. We interpret our results within the framework of pure thermally excited magnons in YIG based on the concept of temperature-dependent effective magnon propagation length, which can explain our results. Beyond the bulk properties, we identify the interfaces as a new major contributor to the temperature dependence of the LSSE signal. A significant change in the LSSE peak position is observed by choosing different capping layers. By TEM analysis of the interfaces, we show that the interfacial structure of YIG/metal films changes depending on the metal layer on top. This change of the YIG termination can be correlated with the amplitude and temperature dependence of the measured SSE signal. Thus, our results reveal that the temperature dependence of the LSSE is not solely governed by the magnetic material itself, as previously assumed, but is, in particular, influenced by the interface between the metal capping layer and ferromagnets, opening new paths to engineering the LSSE signals.

\section{ACKNOWLEDGMENTS}

The authors would like to express their thanks to Dr. Alexander Serga and Professor Burkard Hillebrands at TU Kaiserslautern for providing a portion of the LPE YIG samples and to Professor Gerrit E. W. Bauer, Dr. Joe Barker, Professor Ulrich Nowak, Professor R. Duine, and Dr. Sebastian T. B. Goennenwein for valuable discussions. This work was supported by Deutsche Forschungsgemeinschaft (DFG) SPP 1538 "Spin Caloric Transport," the Graduate School of Excellence Materials Science in Mainz (MAINZ), the EU projects (IFOX, NMP3-LA-2012246102, INSPIN FP7-ICT-2013-X 612759, MASPIC ERC-2007-StG 208162), DAAD Spintronics network (SpinNet), project No. 56268455, and Transregional Collaborative Research Center (SFB/ TRR) 173 "Spin+X-Spin its collective environment", and through the provision of a fellowship to D. A. M., the Engineering and Physical Sciences Research Council of the UK (EP/I00419X/1). During the completion of this manuscript, E.-J. G. was supported by the Laboratory Directed Research and Development (LDRD) Program of Oak Ridge National Laboratory, managed by UT-Battelle, LLC, for the U.S. DOE.

Note added.-Recently, Cornelissen and van Wees [36], Kikkawa et al. [37], and Jin et al. [38] reported related 
results of substantial field suppressions of the LSSE signals, supporting our results. Additionally, the theories of Diniz and Costa [51] and Cornelissen et al. [52] have recently reproduced our temperature and thickness dependence of the LSSE results, corroborating our experiments and extending the explanation of our observations. Moreover, we note that Prakash et al. [53] have shown interface and bulk effects in the spin Seebeck effect by inserting an antiferromagnetic $\mathrm{NiO}$ layer between the $\mathrm{Pt}$ and YIG. They argue that a thin NiO layer leads to a different temperature dependence of spin Seebeck signals, which is in a good agreement with our results. However, note that in their work, the bulk $\mathrm{NiO}$ properties also influence, in addition to the interface effects that we study here, the spin transport and the resulting measured signals.

\section{APPENDIX A: SURFACE CHARACTERISTICS OF YIG FILMS AND HMS/YIG INTERFACES}

The surface characterization of the LPE YIG samples we use in the present study is carried out by AFM. Figure 6(a) shows a typical AFM image of a 50- $\mu \mathrm{m}$-thick YIG film, which reveals a smooth surface with rms roughness of $0.34 \mathrm{~nm}$ over an area of $2.5 \times 2.5 \mu \mathrm{m}^{2}$. All the other YIG samples also show a similar surface smoothness with rms roughness below $0.5 \mathrm{~nm}$. The interfacial smoothness is checked using x-ray reflectivity. Figures 6(b)-6(d) show the XRR interference of three HM/YIG hybrid structures. The fits to the XRR are in good agreement with the experimental scans, from which we can extract the thickness $(t)$ of each layer and the interfacial roughness. The thickness is estimated to be $t_{\mathrm{Pt}} \sim 5.5 \mathrm{~nm}, t_{\mathrm{Pd}} \sim 5.6 \mathrm{~nm}$, and $t_{\mathrm{Cu}} \sim 1.9 \mathrm{~nm}$, respectively, in a good agreement with the STEM results (Fig. 5). The interfacial roughness is calculated to be $0.34,0.52$, and $0.54 \mathrm{~nm}$ for the $\mathrm{Pt} / \mathrm{YIG}$, the $\mathrm{Pd} / \mathrm{YIG}$, and the $\mathrm{Pt} / \mathrm{Cu} / \mathrm{YIG}$ interfaces, respectively. The XRR of the $\mathrm{AlO}_{x} / \mathrm{W} / \mathrm{YIG}$ hybrid shows a rapid decay of signal due to the thick $\mathrm{AlO}_{x}$ protection layer on top of W. To determine the thickness of the $\mathrm{W}$ layer, alternatively, we grow a $\mathrm{W}$ layer with the same thickness on sapphire. The fit to the XRR indicates the thickness of W is $\sim 5.2 \mathrm{~nm}$ (results are not shown). Our surface and interface characteristics indicate the negligible role in the pronounced differences between temperature dependences of the LSSE.

\section{APPENDIX B: DETERMINATION OF TEMPERATURE DIFFERENCE ACROSS THE BILAYERS}

Previous works $[5-7,10,12,15-18]$ adopted the temperature difference $(\Delta T)$ across the hybrid structure from the thermocouples attached to the upper and lower copper plates. Inevitably, the differences in the thermal transport between each electronic component and the samples lead to an uncertainty for the precise determination of the real $\Delta T$. In our experimental setup, the HM detection strips on the top and ground surfaces of the samples can be utilized as resistance-temperature sensors to determine the $\Delta T$ across the HM/YIG hybrids. Figure 7 shows the calibrated $\Delta T$ across the $\mathrm{Pt} / \mathrm{Cu} / \mathrm{YIG}$ hybrid structure as a function of temperature under the heating current of 7 and $9 \mathrm{~mA}$ applied to the resistive heater. $\Delta T$ is not a constant value across the whole temperature range. Instead, it varies with temperature, depending on the cooling rate of the cryostat and the heating power of the resistive heater. The temperature-dependent LSSE coefficients of each sample are calculated by considering the individual $\Delta T$ as a function
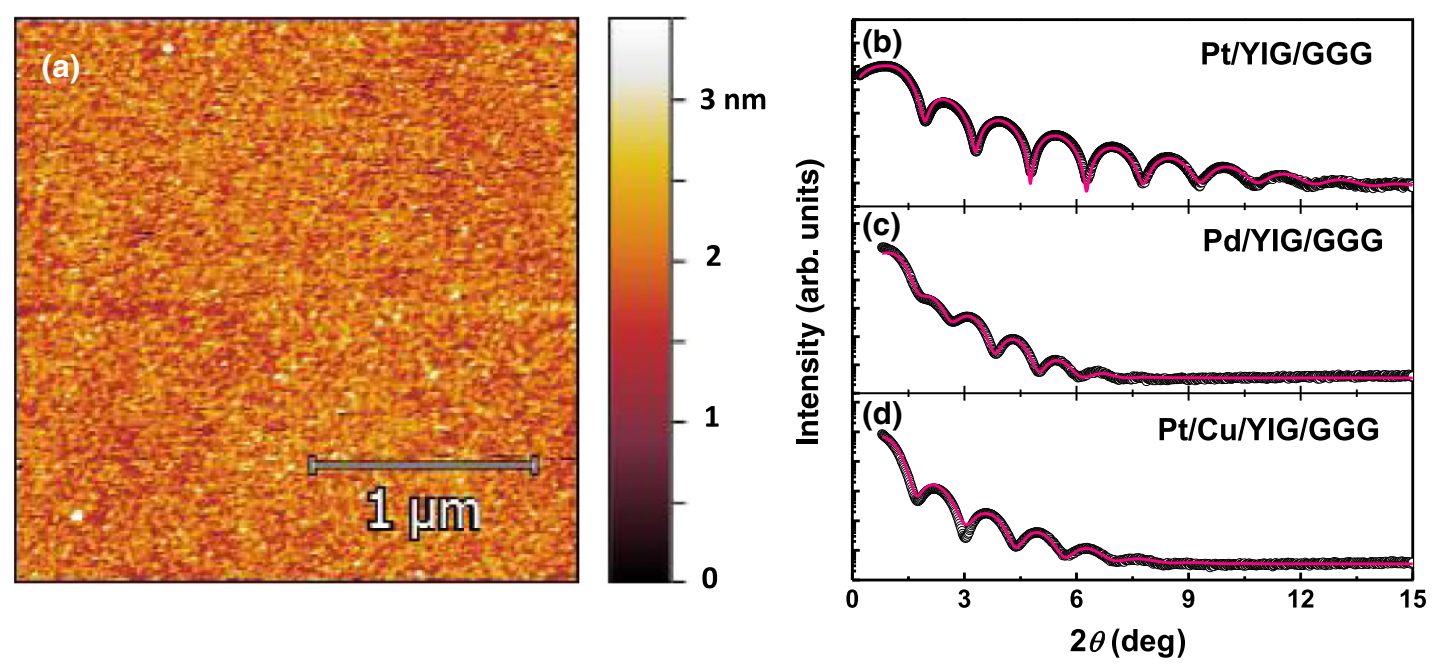

FIG. 6. (a) AFM image of a 50- $\mu$ m-thick LPE YIG film over an area of $2.5 \times 2.5 \mu \mathrm{m}^{2}$ with a rms roughness of $0.34 \mathrm{~nm}$. XRR curves of (b) Pt/YIG, (c) Pd/YIG, and (d) Pt/Cu/YIG hybrid structures are illustrated. The black open symbols are the experimental data, while the red lines are the fitting curves. 


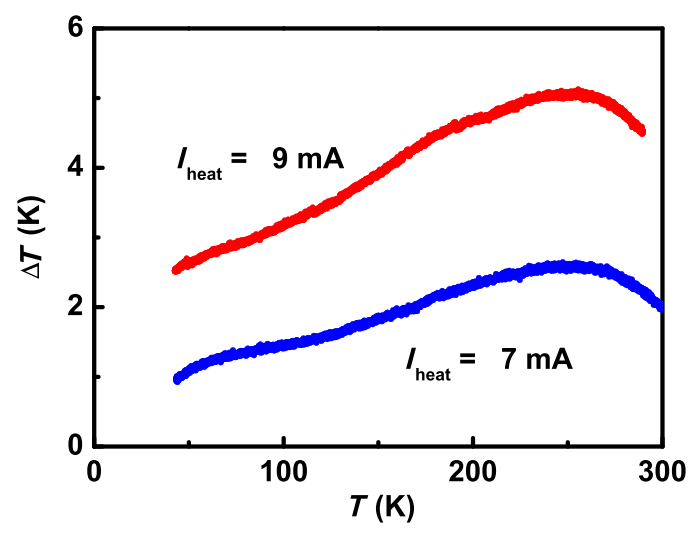

FIG. 7. The calculated $\Delta T$ across the $\mathrm{Pt} / \mathrm{Cu} / \mathrm{YIG}$ hybrid structure as a function of system temperature. Two curves are illustrated under heating currents of 7 and $9 \mathrm{~mA}$, respectively.

of temperature. The temperature used in the present work is the temperature obtained from the Pt strip on the YIG surface, providing the best possible determination of the actual system temperature.

[1] K. Uchida, S. Takahashi, K. Harii, J. Ieda, W. Koshibae, K. Ando, S. Maekawa, and E. Saitoh, Observation of the Spin Seebeck Effect, Nature (London) 455, 778 (2008).

[2] C. M. Jaworski, J. Yang, S. Mack, D. D. Awschalom, J. P. Heremans, and R.C. Myers, Observation of the SpinSeebeck Effect in a Ferromagnetic Semiconductor, Nat. Mater. 9, 898 (2010).

[3] K. Uchida, J. Xiao, H. Adachi, J. Ohe, S. Takahashi, J. Ieda, T. Ota, Y. Kajiwara, H. Umezawa, H. Kawai, G. E. W. Bauer, S. Maekawa, and E. Saitoh, Spin Seebeck Insulator, Nat. Mater. 9, 894 (2010).

[4] C. M. Jaworski, R. C. Myers, E. Johnston-Halperin, and J. P. Heremans, Giant Spin Seebeck Effect in a Non-Magnetic Material, Nature (London) 487, 210 (2012).

[5] T. Kikkawa, K. Uchida, Y. Shiomi, Z. Qiu, D. Hou, D. Tian, H. Nakayama, X.-F. Jin, and E. Saitoh, Longitudinal Spin Seebeck Effect Free from the Proximity Nernst Effect, Phys. Rev. Lett. 110, 067207 (2013).

[6] K. Uchida, H. Adachi, T. Ota, H. Nakayama, S. Maekawa, and E. Saitoh, Observation of Longitudinal Spin-Seebeck Effect in Magnetic Insulators, Appl. Phys. Lett. 97, 172505 (2010).

[7] A. Kehlberger, U. Ritzmann, D. Hinzke, Er-Jia Guo, J. Cramer, G. Jakob, M. C. Onbasli, D. H. Kim, C. A. Ross, M. B. Jungfleisch, B. Hillebrands, U. Nowak, and M. Kläui, Length Scale of the Spin Seebeck Effect, Phys. Rev. Lett. 115, 096602 (2015).

[8] S. Y. Huang, X. Fan, D. Qu, Y. P. Chen, W. G. Wang, J. Wu, T. Y. Chen, J. Q. Xiao, and C. L. Chien, Transport Magnetic Proximity Effects in Platinum, Phys. Rev. Lett. 109, 107204 (2012).

[9] C. M. Jaworski, J. Yang, S. Mack, D. D. Awschalom, R. C. Myers, and J. P. Heremans, Spin-Seebeck Effect: A Phonon
Driven Spin Distribution, Phys. Rev. Lett. 106, 186601 (2011).

[10] H. Adachi, K. Uchida, E. Saitoh, J. Ohe, S. Takahashi, and S. Maekawa, Gigantic Enhancement of Spin Seebeck Effect by Phonon Drag, Appl. Phys. Lett. 97, 252506 (2010).

[11] J. Xiao, G. E. W. Bauer, K. Uchida, E. Saitoh, and S. Maekawa, Theory of Magnon-Driven Spin Seebeck Effect, Phys. Rev. B 81, 214418 (2010).

[12] K. Uchida, T. Ota, H. Adachi, J. Xiao, T. Nonaka, Y. Kaijiwara, G. E. W. Bauer, S. Maekawa, and E. Saitoh, Thermal Spin Pumping and Magnon-Phonon-Mediated Spin-Seebeck Effect, J. Appl. Phys. 111, 103903 (2012).

[13] K. Uchida, H. Adachi, T. An, T. Ota, M. Toda, B. Hillebrands, S. Maekawa, and E. Saitoh, Long-Range Spin Seebeck Effect and Acoustic Spin Pumping, Nat. Mater. 10, 737 (2011).

[14] S. R. Boona and J. P. Heremans, Magnon Thermal Mean Free Path in Yttrium Iron Garnet, Phys. Rev. B 90, 064421 (2014).

[15] Z. Qiu, D. Hou, K. Uchida, and E. Saitoh, Influence of Interface Condition on Spin-Seebeck Effects, J. Phys. D 48, 164013 (2015).

[16] A. Aqeel, I. J. Vera-Marun, B. J. van Wees, and T. T. M. Palstra, Surface Sensitivity of the Spin Seebeck Effect, J. Appl. Phys. 116, 153705 (2014).

[17] Z. Qiu, K. Ando, K. Uchida, Y. Kajiwara, R. Takahashi, H. Nakayama, T. An, Y. Fujikawa, and E. Saitoh, Spin Mixing Conductance at a Well-Controlled Platinum/Yttrium Iron Garnet Interface, Appl. Phys. Lett. 103, 092404 (2013).

[18] K. Uchida, T. Kikkawa, A. Miura, J. Shiomi, and E. Saitoh, Quantitative Temperature Dependence of Longitudinal Spin Seebeck Effect at High Temperatures, Phys. Rev. X 4, 041023 (2014).

[19] S. M. Wu, J. E. Pearson, and A. Bhattacharya, Paramagnetic Spin Seebeck Effect, Phys. Rev. Lett. 114, 186602 (2015).

[20] S. M. Wu, W. Zhang, A. KC, P. Borisov, J. E. Pearson, J. S. Jiang, D. Lederman, A. Hoffmann, and A. Bhattacharya, Antiferromagnetic Spin Seebeck Effect, Phys. Rev. Lett. 116, 097204 (2016).

[21] S. Seki, T. Ideue, M. Kubota, Y. Kozuka, R. Takagi, M. Nakamura, Y. Kaneko, M. Kawasaki, and Y. Tokura, Thermal Generation of Spin Current in an Antiferromagnet, Phys. Rev. Lett. 115, 266601 (2015).

[22] M. Schmid, S. Srichandan, D. Meier, T. Kuschel, J.-M. Schmalhorst, M. Vogel, G. Reiss, C. Strunk, and C. H. Back, Transverse Spin Seebeck Effect versus Anomalous and Planar Nernst Effects in Permalloy Thin Films, Phys. Rev. Lett. 111, 187201 (2013).

[23] K. Demasius, T. Phung, W. Zhang, B. P. Hughes, S.-H Yang, A. Kellock, W. Han, A. Pushp, and S. S. P. Parkin, Enhanced Spin-Orbit Torques by Oxygen Incorporation in Tungsten Films, Nat. Commun. 7, 10644 (2016).

[24] L. Wang, R. J. H. Wesselink, Y. Liu, Z. Yuan, K. Xia, and P. J. Kelly, Giant Room Temperature Interface Spin Hall and Inverse Spin Hall Effects, Phys. Rev. Lett. 116, 196602 (2016). 
[25] B. L. Zink, M. Manno, L. O’Brien, J. Lotze, M. Weiler, D. Bassett, S. J. Mason, S. T. B. Goennenwein, M. Johnson, and C. Leighton, Efficient Spin Transport through Native Oxides of Nickel and Permalloy with Platinum and Gold Overlayers, Phys. Rev. B 93, 184401 (2016).

[26] A. Hojem, D. Wesenberg, and B. L. Zink, Thermal Spin Injection and Interface Insensitivity in Permalloy/Aluminum Metallic Non-Local Spin Valves, arXiv:1602.03859.

[27] J. A. Hunt and D. B. Williams, Electron Energy-Loss Spectrum-Imaging, Ultramicroscopy 38, 47 (1991).

[28] J. Scott, P. J. Thomas, M. MacKenzie, S. McFadzean, J. Wilbrink, A. J. Craven, and W. A. P. Nicholson, NearSimultaneous Dual Energy Range EELS Spectrum Imaging, Ultramicroscopy 108, 1586 (2008).

[29] M. Isasa, E. Villamor, L. E. Hueso, M. Gradhand, and F. Casanova, Temperature Dependence of Spin Diffusion Length and Spin Hall Angle in Au and Pt, Phys. Rev. B 91, 024402 (2015).

[30] C. Euler, P. Hołuj, T. Langner, A. Kehlberger, V. I. Vasyuchka, M. Kläui, and G. Jakob, Thermal Conductance of Thin Film YIG Determined Using Bayesian Statistics, Phys. Rev. B 92, 094406 (2015).

[31] U. Ritzmann, D. Hinzke, and U. Nowak, Propagation of Thermally Induced Magnonic Spin Currents, Phys. Rev. B 89, 024409 (2014).

[32] U. Ritzmann, D. Hinzke, A. Kehlberger, E. J. Guo, M. Kläui, and U. Nowak, Magnetic Field Control of the Spin Seebeck Effect, Phys. Rev. B 92, 174411 (2015).

[33] M. B. Jungfleisch, A. V. Chumak, A. Kehlberger, V. Lauer, D. H. Kim, M. C. Onbasli, C. A. Ross, M. Kläui, and B. Hillebrands, Thickness and Power Dependence of the SpinPumping Effect in $\mathrm{Y}_{3} \mathrm{Fe}_{5} \mathrm{O}_{12} / \mathrm{Pt}$ Heterostructures Measured by the Inverse Spin Hall Effect, Phys. Rev. B 91, 134407 (2015).

[34] E. G. Spencer and R. C. LeCraw, Ferromagnetic Relaxation in Yttrium-Iron Garnet and the Relation to Applications, Proc. IEE 109, 66 (1962).

[35] L. J. Cornelissen, J. Liu, R. A. Duine, J. Ben Youssef, and B. J. van Wees, Long Distance Transport of Magnon Spin Information in a Magnetic Insulator at Room Temperature, Nat. Phys. 11, 1022 (2015).

[36] L. J. Cornelissen and B. J. van Wees, Magnetic Field Dependence of the Magnon Spin Diffusion Length in the Magnetic Insulator Yttrium Iron Garnet, Phys. Rev. B 93, 020403(R) (2016).

[37] T. Kikkawa, K. Uchida, S. Daimon, Z. Qiu, Y. Shiomi, and E. Saitoh, Critical Suppression of Spin Seebeck Effect by Magnetic Fields, Phys. Rev. B 92, 064413 (2015).

[38] H. Jin, S. R. Boona, Z. Yang, R. C. Myers, and J. P. Heremans, Effect of the Magnon Dispersion on the Longitudinal Spin Seebeck Effect in Yttrium Iron Garnets, Phys. Rev. B 92, 054436 (2015).

[39] M. Weiler, M. Althammer, M. Scherier, J. Lotze, M. Pernpeintner, S. Meyer, H. Huebl, R. Gross, A. Kamra,
J. Xiao, Y.-T. Chen, H. Jiao, G. E. W. Bauer, and S. T. B. Goennenwein, Experimental Test of the Spin Mixing Interface Conductivity Concept, Phys. Rev. Lett. 111, 176601 (2013).

[40] J. Ren, Predicted Rectification and Negative Differential Spin Seebeck Effect at Magnetic Interfaces, Phys. Rev. B 88, 220406(R) (2013).

[41] H. L. Wang, C. H. Du, Y. Pu, R. Adur, P. C. Hammel, and F. Y. Yang, Scaling of Spin Hall Angle in 3d, 4d, and 5d Metals from $\mathrm{Y}_{3} \mathrm{Fe}_{5} \mathrm{O}_{12} /$ Metal Spin Pumping, Phys. Rev. Lett. 112, 197201 (2014).

[42] Y. Wang, P. Deorani, X. Qiu, J. H. Kwon, and H. Yang, Determination of Intrinsic Spin Hall Angle in Pt, Appl. Phys. Lett. 105, 152412 (2014).

[43] J. Bass and W. P. Prat, Determination of Penetration Depth of Transverse Spin Current in Ferromagnetic Metals by Spin Pumping, J. Phys. Condens. Matter 19, 183201 (2007).

[44] Z. Tang, Y. Kitamura, E. Shikoh, Y. Ando, T. Shinjo, and M. Shiraishi, Temperature Dependence of Spin Hall Angle of Palladium, Appl. Phys. Express 6, 083001 (2013).

[45] H. Kurt, R. Loloee, K. Eid, W. P. Pratt, and J. Bass, Spin-Memory Loss at $4.2 \mathrm{~K}$ in Sputtered Pd and Pt and at $\mathrm{Pd} / \mathrm{Cu}$ and $\mathrm{Pt} / \mathrm{Cu}$ Interfaces, Appl. Phys. Lett. 81, 4787 (2002).

[46] F. J. Jedema, M. S. Nijboer, A. T. Filip, and B. J. van Wees, Spin Injection and Spin Accumulation in AllMetal Mesoscopic Spin Valves, Phys. Rev. B 67, 085319 (2003).

[47] T. Kimura, Y. Otani, T. Sato, S. Takahashi, and S. Maekawa, Room-Temperature Reversible Spin Hall Effect, Phys. Rev. Lett. 98, 156601 (2007).

[48] S. R. Etesami, L. Chotorlishvili, and J. Berakdar, Spectral Characteristics of Time Resolved Magnonic Spin Seebeck Effect, Appl. Phys. Lett. 107, 132402 (2015).

[49] T. Tashiro, R. Takahashi, Y. Kajiwara, K. Ando, H. Nakayama, T. Yoshino, D. Kikuchi, and E. Saitoh, Thickness Dependence of Spin Pumping at YIG/Pt Interface, Proc. SPIE Int. Soc. Opt. Eng. 8461, 846106 (2012).

[50] O. Mosendz, V. Vlaminck, J. E. Pearson, F. Y. Fradin, G. E. W. Bauer, S. D. Bader, and A. Hoffmann, Detection and Quantification of Inverse Spin Hall Effect from Spin Pumping in Permalloy/Normal Metal Bilayers, Phys. Rev. B 82, 214403 (2010).

[51] I. Diniz and A. J. Costa, Microscopic Origin of Subthermal Magnons and the Spin Seebeck Effect, New J. Phys. 18, 052002 (2016).

[52] L. J. Cornelissen, K. J. H. Peters, G. E. W. Bauer, R. A. Duine, and B. J. van Wees, Magnon Spin Transport Driven by the Magnon Chemical Potential in a Magnetic Insulator, arXiv:1604.03706 [Phys. Rev. B (to be published)].

[53] A. Prakash, J. Brangham, F. Yang, and J. P. Heremans, Spin Seebeck Effect through Antiferromagnetic NiO, arXiv: 1604.08659. 